\title{
REPRODUCCIÓN GENERACIONAL DEL MALTRATO INFANTIL
}

\author{
Lupe Garcia A. ${ }^{1}$, Oswaldo Orellana M., Ricardo Pomalaya V., Elisa Yanac R.,Carmela Malaver S., Edgar \\ Herrera F. , Noemi Sotelo L., Lilia Campos C., Lidia Sotelo L., Daphne Orellana G., Katherine Velasquez M. \\ Universidad Nacional Mayor de San Marcos, Lima, Perú \\ (RECIBIDO EL 17/09/2008, ACEPTADO EL 18/12/2008)
}

\begin{abstract}
RESUMEN
El objetivo de la presente investigación es el estudio de la reproducción del maltrato en dos generaciones, la de los padres y de los hijos, y el establecimiento de la relación entre la historia de maltrato infantil con el potencial de maltrato.

La muestra está conformada por 441 estudiantes universitarios y 303 padres que aceptaron responder al cuestionario de recuerdos de maltrato. Los instrumentos utilizados fueron el Cuestionario de historia infantil, adaptado para fines de la presente investigación, y el Inventario de potencial de maltrato infantil (Milner, J. 1977), adaptado por De Paúl, Arruabarrena, Múgica y Milner, 1999 en España .

Los resultados muestran que tanto en padres e hijos el maltrato emocional es más frecuente seguido del maltrato físico y abuso sexual, comparando las dos generaciones, la de los padres presenta una mayor incidencia de maltrato. Asimismo el potencial de maltrato está asociado significativamente con la historia de maltrato físico y emocional y el maltrato a los hijos está asociado, significativamente, a una historia de maltrato de los padres; sin embargo, también se reporta que el círculo de reproducción generacional del maltrato no es absoluto.
\end{abstract}

Palabras clave: Maltrato, reproducción generacional, potencial de maltrato, recuerdos de maltrato.

\begin{abstract}
The objective of this research is the study of the abuse rising in two generations, of parents and children and establishing the relationship between background child's abuse with the potential abuse.

The sample is comprised of 441 students and 303 parents who agreed to answer the Memories of Abuse Questionnaire. The used instruments were the Child History Questionnaire adapted for the purpose of this research, the Inventory of Potential Child Abuse (Milner, J. 1977), adapted by De Paul, Arruabarrena, Múgica and Milner, 1999 in Spain.

The results show that both of them (parent and child) the emotional abuse is more frequently followed by physical and sexual abuse, comparing the two generations, the parents show a higher prevalence of abuse. Also the potential for abuse is significantly associated with history of physical abuse and emotional abuse and the children`s abuse is significantly
\end{abstract}

1 Docente Principal, Ex Decana y Directora de la Unidad de Post Grado de la Facultad de Psicología, UNMSM.

E-mail: Igarciape@yahoo.es 
associated with a parents abuse history, however also reported that the reproductive cycle of generational abuse is not all.

Keywords: Abuse, generational reproduction, potential for abuse, memories of abuse.

\section{INTRODUCCIÓN}

El maltrato infantil es una realidad dolorosa porque afecta a quienes no pueden defenderse del mismo, los ejecutores son los padres que se supone deben garantizar el cuidado y bienestar del menor; en esta dinámica se generan relaciones de alto riesgo para la socialización de menores.

Se supone que el hogar es el lugar más seguro para las personas, sin embargo, en el caso del maltrato, se convierten en lugares peligrosos no solo para el menor sino también para los adultos que maltratan o que son espectadores pasivos de lo que sucede.

Hasta la década de los 70 las hipótesis más fuertes respecto a la búsqueda de explicación de las razones que conducen a los padres a maltratar a sus hijos, fue que ellos a su vez arrastraban una historia de maltrato en su infancia y lo único que hacían era reproducir los modelos recibidos, cerrándose el círculo de la violencia, sin que haya posibilidad de escapatoria.

A partir de la década del 80-90 distintos investigadores (Kaufman y Zigler, 1987, 1989; Burgess y Youngblade, 1988; Widom,1989; Knutson, 1995) evalúan críticamente los resultados de las investigaciones previas, observando la existencia de errores metodológicos en las mismas, la más resaltante es la referida a las muestras con las cuales se trabajó: todos eran padres de hijos maltratados, los resultados daban cuenta que éstos al maltratar a sus hijos estaban repitiendo la historia vivida, pero no se tomaba en cuenta grupos de comparación: niños cuyos padres no los maltrataban, aún cuando ellos si tuvieron una historia de maltrato infantil.

Reconocidos los errores metodológicos, se replantearon las investigaciones arrojando resultados contradictorios, resultados que apoyan la hipótesis de la trasmisión intergeneracional del maltrato (Smith y Thornberry, 1995, Zingraff et al 1993 citados por E. Gomez y J. De Paúl, 2003) y otros que afirman que la historia de maltrato no incrementa significativamente el maltrato posterior.

Para el presente estudio, se trabajó con dos generaciones: padres e hijos, la primera aproximación fue con los hijos quienes, a nivel retrospectivo, evocaron situaciones de presencia o no de tres tipos de maltrato: maltrato físico, abuso sexual y maltrato emocional, con lo que se identificó a los padres que maltratan de aquellos que no lo hacen, asimismo se aplicó el cuestionario de potencial de maltrato a todos los participantes de la muestra, lo que permitió establecer relaciones entre la historia de maltrato y el potencial para hacerlo en el futuro. En una segunda fase, los padres a su vez evocaron su historia infantil en relación a su condición de niños maltratados o no, en los tres tipos de maltrato considerados; como se puede deducir los padres que por la versión de sus hijos los han maltratado como los que no lo hicieron fueron parte de la muestra para evitar el sesgo de entrevistar solo a los padres maltratadores y en el entendido que los padres que no han maltratado a sus hijos puedan tener una historia de maltrato.

Las interrogantes que nos planteamos son las siguientes: 
- ¿Existen diferencias de género en los tres tipos de maltrato: maltrato físico, abuso sexual y maltrato emocional?

- ¿Cuáles son las relaciones entre la historia de maltrato y el potencial de maltrato en la generación de hijos?

- ¿Existe reproducción generacional del maltrato en quienes fueron maltratados en su infancia?

\section{MÉTODO}

\section{Objetivos}

- $\quad$ Establecer las diferencias de género en la exposición a los tres tipos de maltrato: maltrato físico, abuso sexual y maltrato emocional

- Establecer las relaciones entre la historia de maltrato y el potencial de maltrato en la generación de hijos

- Establecer las relaciones entre la historia de maltrato en la infancia y la reproducción del maltrato con los hijos.

\section{Participantes}

En la Tabla N. ${ }^{o} 1$ se presenta la distribución de la muestra de acuerdo al género. El número total de hijos es de 441 estudiantes universitarios, de los cuales 162 son hombres y 279 mujeres.

Tabla N. ${ }^{\circ}$ 1. Muestra de hijos según género.

\begin{tabular}{lc}
\hline & Frecuencia \\
\hline Masculino & 162 \\
Femenino & 279 \\
Total & 441 \\
\hline
\end{tabular}

En la Tabla N. ${ }^{\circ} 2$ se presenta la muestra de padres según género, el total de la muestra es de 303 , de los cuales 85 son del sexo masculino y 218 de sexo femenino.

Tabla N. ${ }^{\circ}$ 2. Muestra de padres según género.

\begin{tabular}{lc}
\hline \multicolumn{1}{c}{ Sexo } & Total \\
\hline Masculino & 85 \\
Femenino & 218 \\
Total & 303 \\
\hline
\end{tabular}




\section{Diseño de investigación}

El diseño de investigación es descriptivo comparativo correlacional, porque caracteriza retrospectivamente la historia de maltrato y prospectivamente el potencial del mismo; comparativo porque analiza las diferencias en dos generaciones: padres e hijos y correlacional porque establece relaciones entre la historia de maltrato y la reproducción del maltrato con los hijos, así como la historia de maltrato con el potencial de maltrato.

\section{Instrumentos de recolección de datos}

Para la evaluación retrospectiva del maltrato infantil, se utilizó el Cuestionario de historia infantil, adaptado al español por Gómez, E. y De Paúl, J. (2003), y que a su vez fue adaptado en la presente investigación considerando: el maltrato físico, emocional y abuso sexual.

Para la evaluación prospectiva del maltrato, se utilizó el Inventario de potencial de maltrato infantil (Milner, 1977), que evalúa el riesgo de maltrato infantil futuro, adaptado por De Paúl, Arruabarrena, Múgica y Milner (1999) en España.

\section{Procedimientos de recolección de datos}

En una primera fase se aplicó el cuestionario de historia infantil y el inventario de potencial de maltrato infantil a 441 estudiantes universitarios de Lima. En una segunda fase se procedió a solicitar la colaboración a los padres para participar en la investigación, todos los padres que aceptaron fueron evaluados con el cuestionario de historia infantil. En esta fase participaron los padres que según los recuerdos de sus hijos los han maltratado y los que no han maltratado, para evitar el error metodológico de solo considerar a los maltratadores y no tener a un grupo de contraste.

\section{RESULTADOS}

En la Tabla N. ${ }^{\circ} 3$ se presenta la frecuencia de maltrato en los hijos, considerando el maltrato físico, abuso sexual y maltrato emocional. El maltrato emocional es más frecuente $(33,2 \%)$, seguida de maltrato físico $(15,7 \%)$ y abuso sexual $(1,6 \%)$.

Tabla N. ${ }^{0}$ 3. Frecuencia de los tipos de maltrato en hijos.

\begin{tabular}{lccccccc}
\hline & \multicolumn{2}{c}{ Maltrato físico } & \multicolumn{2}{c}{ Abuso sexual } & \multicolumn{2}{c}{ Maltrato emocional } \\
& $\mathbf{N}^{\mathbf{0}}$ de hijos & $\mathbf{\%}$ & $\mathbf{N}^{\mathbf{0}}$ de hijos & $\mathbf{\%}$ & $\mathbf{N}^{\mathbf{0}}$ de hijos & \% \\
\hline Sin maltrato & 371 & $84,3 \%$ & 434 & $98,4 \%$ & 294 & $66,8 \%$ \\
Con maltrato & 69 & $15,7 \%$ & 7 & $1,6 \%$ & 146 & $33,2 \%$ \\
Total & $\mathbf{4 4 0}$ & $\mathbf{1 0 0 , 0 \%}$ & $\mathbf{4 4 1}$ & $\mathbf{1 0 0 , 0 \%}$ & $\mathbf{4 4 0}$ & $\mathbf{1 0 0 , 0 \%}$ \\
\hline
\end{tabular}

En la Tabla N. ${ }^{\circ} 4$ se presenta la frecuencia de maltrato en los padres, considerando el maltrato físico, abuso sexual y maltrato emocional. El maltrato emocional es más frecuente $(40,7 \%)$, seguida de maltrato físico $(32,6 \%)$ y abuso sexual $(1,7 \%)$. 
Tabla N. ${ }^{\circ}$ 4. Frecuencia de los tipos de maltrato en los padres.

\begin{tabular}{|c|c|c|c|c|c|c|}
\hline & \multicolumn{2}{|c|}{ Maltrato físico padres } & \multicolumn{2}{|c|}{ Abuso sexual padres } & \multicolumn{2}{|c|}{$\begin{array}{c}\text { Maltrato emocional } \\
\text { padres }\end{array}$} \\
\hline & $\begin{array}{l}\text { N. }{ }^{\circ} \text { de } \\
\text { padres }\end{array}$ & $\%$ & $\begin{array}{l}\text { N. }{ }^{\circ} \text { de } \\
\text { padres }\end{array}$ & $\%$ & $\begin{array}{l}\text { N. }{ }^{\circ} \text { de } \\
\text { padres }\end{array}$ & $\%$ \\
\hline Sin maltrato & 203 & $67,4 \%$ & 297 & $98,3 \%$ & 179 & $59,3 \%$ \\
\hline Con maltrato & 98 & $32,6 \%$ & 5 & $1,7 \%$ & 123 & $40,7 \%$ \\
\hline Total & 301 & $100,0 \%$ & 302 & $100,0 \%$ & 302 & $100,0 \%$ \\
\hline
\end{tabular}

En la Tabla N. ${ }^{\circ} 5$ se presenta los resultados de las diferencias de género en la muestra de hijos con y sin recuerdos de maltrato físico; los que registran recuerdos de maltrato físico son 69 y sin recuerdos de maltrato 371. De acuerdo a la medida de asociación, no existe relación significativa entre los recuerdos de maltrato físico y el género $\mathrm{p}>0.05$.

Tabla N. ${ }^{0}$ 5. Diferencias de género en hijos con y sin recuerdos de maltrato físico.

\begin{tabular}{cccccc}
\hline & & \multicolumn{2}{c}{ Sexo } & Total \\
& & & Masculino & Femenino & \\
\hline Maltrato físico & Sin maltrato & Recuento & 132 & 239 & 371 \\
& & \% de Maltrato físico & $35.6 \%$ & $64.4 \%$ & $100.0 \%$ \\
& \multirow{2}{*}{ Con maltrato } & Recuento & 29 & 40 & 69 \\
& \% de Maltrato físico & $42.0 \%$ & $58.0 \%$ & $100.0 \%$ \\
& Total & Recuento & 161 & 279 & 440 \\
& \% de Maltrato físico & $36.6 \%$ & $63.4 \%$ & $100.0 \%$ \\
\hline
\end{tabular}

Chi-cuadrado de Pearson: 1.043(b) P > 0.05 no existe relación significativa.

En la Tabla N. ${ }^{\circ} 6$ se presenta los resultados de las diferencias de género en la muestra de hijos con y sin recuerdos de abuso sexual, los que registran recuerdos de abuso sexual son 7 y 434 sin recuerdos de maltrato. De acuerdo a la medida de asociación, no existe relación significativa entre los recuerdos de abuso sexual y el género. $\mathrm{p}>0.05$.

Tabla N. ${ }^{\circ}$ 6. Diferencias de género en sujetos con y sin recuerdos de abuso sexual.

\begin{tabular}{cclccc}
\hline & & \multicolumn{2}{c}{ Sexo } & Total \\
& & & Masculino & Femenino & \\
\hline Abuso sexual & Sin maltrato & Recuento & 161 & 273 & 434 \\
& & $\%$ de Abuso sexual & $37.1 \%$ & $62.9 \%$ & $100.0 \%$ \\
& \multirow{2}{*}{ Con maltrato } & Recuento & 1 & 6 & 7 \\
& \% de Abuso sexual & $14.3 \%$ & $85.7 \%$ & $100.0 \%$ \\
& Recuento & 162 & 279 & 441 \\
& \% de Abuso sexual & $36.7 \%$ & $63.3 \%$ & $100.0 \%$ \\
\hline
\end{tabular}

Chi-cuadrado de Pearson: 1.542(b) P > 0.05, no existe relación significativa. 
En la Tabla N. ${ }^{\circ} 7$ se presenta los resultados de las diferencias de género en la muestra de hijos con y sin recuerdos de maltrato emocional, los que registran recuerdos de maltrato emocional son 146 y sin recuerdos de maltrato 294. De acuerdo a la medida de asociación, no existe relación significativa entre los recuerdos de maltrato emocional y el género $\mathrm{p}$ $>0.05$.

Tabla N. ${ }^{\circ}$ 7. Diferencias de género en hijos con y sin maltrato emocional.

\begin{tabular}{cclccc}
\hline & & \multicolumn{2}{c}{ Sexo } & \multicolumn{2}{c}{ Total } \\
& & & Masculino & Femenino \\
\hline Maltrato emocional & Sin maltrato & Recuento & 113 & 181 & 294 \\
& & $\%$ de Maltrato emocional & $38,4 \%$ & $61.6 \%$ & $100,0 \%$ \\
& \multirow{2}{*}{ Con maltrato } & Recuento & 49 & 97 & 146 \\
& & $\%$ de Maltrato emocional & $33,6 \%$ & $66,4 \%$ & $100,0 \%$ \\
& & Recuento & 162 & 278 & 440 \\
& & $\%$ de Maltrato emocional & $36,8 \%$ & $63,2 \%$ & $100,0 \%$ \\
\hline
\end{tabular}

Chi-cuadrado de Pearson: .996(b) P > 0.05, no existe relación significativa.

En la Tabla N. ${ }^{\circ} 8$ se presenta la frecuencia y porcentaje de potencial de maltrato en la muestra de hijos. De la muestra total $345(78,2 \%)$ no presentan potencial de maltrato y $96(21,8 \%)$ si presentan potencial de maltrato

Tabla N. ${ }^{\circ}$. Potencial de maltrato en hijos.

\begin{tabular}{cccc}
\hline & & Frecuencia & Porcentaje \\
\hline Válidos & No & 345 & 78.2 \\
& Sí & 96 & 21.8 \\
& Total & 441 & 100.0 \\
\hline
\end{tabular}

En la Tabla N. ${ }^{\circ} 9$ se presenta en la muestra de hijos la relación de recuerdos de maltrato físico y el potencial de maltrato, los resultados del análisis estadístico $\mathrm{P}<0.05$, señalan que existe asociación significativa entre ambas variables.

Tabla N. ${ }^{\circ}$ 9. Recuerdos de maltrato físico y su relación con el potencial de maltrato (hijos).

\begin{tabular}{|c|c|c|c|c|c|}
\hline & & & \multicolumn{2}{|c|}{ Maltrato físico } & \multirow{2}{*}{ Total } \\
\hline & & & Sin maltrato & Con maltrato & \\
\hline \multirow{4}{*}{$\begin{array}{l}\text { Potencial de } \\
\text { maltrato }\end{array}$} & \multirow{2}{*}{ No } & Recuento & 310 & 35 & 345 \\
\hline & & $\%$ de Potencial maltrato & $89,9 \%$ & $10,1 \%$ & $100,0 \%$ \\
\hline & \multirow{2}{*}{ Sí } & Recuento & 61 & 34 & 95 \\
\hline & & $\%$ de Potencial maltrato & $64,2 \%$ & $35,8 \%$ & $100,0 \%$ \\
\hline \multirow{2}{*}{ Total } & & Recuento & 371 & 69 & 440 \\
\hline & & $\%$ de Potencial maltrato & $84,3 \%$ & $15,7 \%$ & $100,0 \%$ \\
\hline
\end{tabular}

Chi-cuadrado de Pearson: 37.048(b) P < 0.05, existe relación significativa. 
En la Tabla N. ${ }^{\circ} 10$ se presenta la relación ente recuerdos de abuso sexual y el potencial de maltrato, los resultados del análisis estadístico $\mathrm{P}>0.05$, señalan que no hay asociación significativa entre ambas variables

Tabla N. ${ }^{\circ}$ 10. Recuerdos de abuso sexual y su relación con el potencial de maltrato (hijos).

\begin{tabular}{|c|c|c|c|c|c|}
\hline & & & \multicolumn{2}{|c|}{ Abuso sexual } & \multirow{2}{*}{ Total } \\
\hline & & & Sin maltrato & Con maltrato & \\
\hline \multirow{4}{*}{$\begin{array}{l}\text { Potencial de } \\
\text { maltrato }\end{array}$} & \multirow{2}{*}{ No } & Recuento & 341 & 4 & 345 \\
\hline & & $\%$ de potencial de maltrato & $98,8 \%$ & $1,2 \%$ & $100,0 \%$ \\
\hline & \multirow{2}{*}{ Sí } & Recuento & 93 & 3 & 96 \\
\hline & & $\%$ de potencial de maltrato & $96,9 \%$ & $3,1 \%$ & $100,0 \%$ \\
\hline \multirow{2}{*}{ Total } & & Recuento & 434 & 7 & 441 \\
\hline & & $\%$ de potencial de maltrato & $98,4 \%$ & $1,6 \%$ & $100,0 \%$ \\
\hline
\end{tabular}

Chi-cuadrado de Pearson: 1.857 (b) P > 0.05, no existe relación significativa.

En la Tabla N. ${ }^{0} 11$ se presenta en la muestra de hijos la relación de recuerdos de maltrato emocional y el potencial de maltrato, los resultados del análisis estadístico $\mathrm{P}<0.05$, señalan que existe asociación significativa entre ambas variables

Tabla N. ${ }^{\circ}$ 11. Recuerdos de maltrato emocional y su relación con el potencial de maltrato (hijos).

\begin{tabular}{cllccc}
\hline & & \multicolumn{2}{c}{ Maltrato emocional } & \multirow{2}{*}{ Total } \\
& & Sin maltrato & Con maltrato & \\
\hline & No & Recuento & 253 & 91 & 344 \\
\multirow{2}{*}{$\begin{array}{c}\text { Potencial de } \\
\text { maltrato }\end{array}$} & \% de Potencial de maltrato & $73,5 \%$ & $26,5 \%$ & $100,0 \%$ \\
& Sí & Recuento & 41 & 55 & 96 \\
& \% de Potencial de maltrato & $42,7 \%$ & $57,3 \%$ & $100,0 \%$ \\
& Recuento & 294 & 146 & 440 \\
& Total & & $66,8 \%$ & $33,2 \%$ & $100,0 \%$ \\
\hline
\end{tabular}

Chi-cuadrado de Pearson: 32.193(b) $P<0.05$, existe relación significativa.

En la Tabla N. ${ }^{\circ} 12$ se presenta la relación de los recuerdos de maltrato físico en la muestra de hijos y padres, los resultados del análisis estadístico $\mathrm{P}<0.05$, señalan que existe asociación significativa entre ambas variables. 
Tabla N. ${ }^{\circ}$ 12. Recuerdos de maltrato físico de los hijos y padres.

\begin{tabular}{cllccc}
\hline & & & \multicolumn{2}{c}{ Maltrato físico padres } & Total \\
& & & Sin maltrato & Con maltrato & \\
\hline \multirow{2}{*}{$\begin{array}{c}\text { Maltrato } \\
\text { físico hijos }\end{array}$} & \multirow{2}{*}{ Sin maltrato } & Recuento & 187 & 69 & 256 \\
& \multirow{2}{*}{ Con maltrato } & \% de Maltrato físico & $73,0 \%$ & $27,0 \%$ & $100,0 \%$ \\
& & Recuento & 16 & 29 & 45 \\
& \multirow{2}{*}{ Total } & Recuento & $35,6 \%$ & $64,4 \%$ & $100,0 \%$ \\
& \% de Maltrato físico & $67,4 \%$ & $32,6 \%$ & $100,0 \%$ \\
\hline
\end{tabular}

Chi-cuadrado de Pearson: . 24.500(b) P $<0.05$, existe relación significativa.

En la Tabla N. ${ }^{\circ} 13$ se presenta la relación de recuerdos de abuso sexual de padres e hijos y las relaciones entre ambas, los resultados del análisis estadístico $\mathrm{P}<0.05$ señalan que existe asociación significativa entre ambas variables.

Tabla N. ${ }^{0}$ 13. Recuerdos de abuso sexual de los hijos y los padres.

\begin{tabular}{lllccc}
\hline & & & \multicolumn{2}{c}{ Abuso sexual padre } & Total \\
& & & Sin maltrato & Con maltrato & \\
\hline \multirow{3}{*}{$\begin{array}{c}\text { Abuso sexual } \\
\text { hijos }\end{array}$} & \multirow{2}{*}{ Sin maltrato } & Recuento & 295 & 4 & 299 \\
& \multirow{2}{*}{ Con maltrato } & R de Abuso sexual & $98,7 \%$ & $1,3 \%$ & $100,0 \%$ \\
& & \% de Abuso sexual & $66,7 \%$ & $33,3 \%$ & $100,0 \%$ \\
& \multirow{2}{*}{ Total } & Recuento & 297 & 5 & 302 \\
& & $\%$ de Abuso sexual & $98,3 \%$ & $1,7 \%$ & $100,0 \%$ \\
\hline
\end{tabular}

Chi-cuadrado de Pearson: $18.675(b) P<0.05$, existe relación significativa.

En la Tabla N. ${ }^{\circ} 14$ se presenta la relación de los recuerdos de maltrato emocional en la muestra de hijos y padres, los resultados del análisis estadístico $\mathrm{P}<0.05$, señalan que existe asociación significativa entre ambas variables.

Tabla N. ${ }^{\circ}$ 14. Recuerdos de maltrato emocional de hijos y padres.

\begin{tabular}{|c|c|c|c|c|c|}
\hline & & & \multicolumn{2}{|c|}{ Maltrato emocional padres } & \multirow{2}{*}{ Total } \\
\hline & & & Sin maltrato & Con maltrato & \\
\hline \multirow{4}{*}{$\begin{array}{c}\text { Maltrato } \\
\text { emocional } \\
\text { hijos }\end{array}$} & \multirow{2}{*}{ Sin maltrato } & Recuento & 141 & 58 & 199 \\
\hline & & $\%$ de Maltrato emocional & $70.9 \%$ & $29.1 \%$ & $100.0 \%$ \\
\hline & \multirow{2}{*}{ Con maltrato } & Recuento & 38 & 64 & 102 \\
\hline & & $\%$ de Maltrato emocional & $37.3 \%$ & $62.7 \%$ & $100.0 \%$ \\
\hline \multirow{2}{*}{\multicolumn{2}{|c|}{ Total }} & Recuento & 179 & 122 & 301 \\
\hline & & $\%$ de Maltrato emocional & $59.5 \%$ & $40.5 \%$ & $100.0 \%$ \\
\hline
\end{tabular}

Chi-cuadrado de Pearson: 31.584(b)P $<0.05$, existe relación significativa. 


\section{ANÁLISIS Y DISCUSIÓN}

En relación a los recuerdos de maltrato en la muestra de padres e hijos se observa una incidencia mayor de maltrato emocional, seguido de maltrato físico y en menor frecuencia el abuso sexual; sin embargo, globalmente, la frecuencia de maltrato en la generación de padres e hijos es diferente, en la generación de padres la incidencia es mayor (40,7\% de maltrato emocional, frente al 33,2\% de hijos); el maltrato físico en los padres es de 32,6\% y la de los hijos es de $15,7 \%$, en abuso sexual en los padres es de $1,7 \%$ y en los hijos de $1,6 \%$, lo que se podría interpretar en relación a la mayor sensibilización respecto a los derechos del niño y una sanción social del maltrato así como una mayor conciencia del rol de padres en las nuevas generaciones; es importante señalar que la mayor diferencia se encuentra en el maltrato físico, observándose una disminución significativa en la generación de hijos.

No se encontró relación significativa en los recuerdos de maltrato físico y el género, sin embargo, las mujeres presentan un mayor porcentaje de maltrato físico (58\%) en relación a los hombres (42\%), lo que indica que la mujer es más vulnerable para ser víctima de maltrato. Igualmente no se encontró relación significativa en los recuerdos de maltrato emocional y el género, en este caso son también las mujeres las que reportan mayores porcentajes $(66,4 \%)$ frente a $33,6 \%$ de hombres.

En relación al potencial de maltrato en la generación de hijos, se encontró que 96 presentan potencial de maltrato, frente a 346 que no lo presentan, cifra que es preocupante debido a que al tratarse de la generación de hijos, son jóvenes que presentan riesgo de ser maltratadores futuros.

En relación a los recuerdos de maltrato físico y el potencial de maltrato, se encuentra una relación significativa entre ambas variables. De los sujetos con potencial de maltrato un alto porcentaje $(64,2 \%)$ no reporta recuerdos de maltrato físico frente a un $35 \%$ que si tiene recuerdos de maltrato físico. Del grupo que no presenta potencial de maltrato, $10,1 \%$ tiene recuerdos de maltrato físico y $89,9 \%$ no tiene recuerdos de maltrato físico, lo que significa que de los sujetos que reportan recuerdos de maltrato un mayor porcentaje presenta potencial de maltrato, aún cuando es importante relevar que existe un porcentaje de sujetos que a pesar de haber sido maltratados físicamente no presentan potencial de maltrato.

Cuando se relaciona los recuerdos de maltrato emocional y el potencial de maltrato se encuentra una relación significativa, en el grupo de sujetos con potencial de maltrato un $57,3 \%$ tiene recuerdos de maltrato emocional y un $42,7 \%$ no registra recuerdos; lo que significaría que alguien puede ser un potencial maltratador sin historia de maltrato.

En los sujetos que no presentan potencial de maltrato el 26,5\% tiene recuerdos de maltrato emocional y un $73,5 \%$ no los tiene; estos datos nos indican que no todo el que sufre maltrato emocional lo reproducirá en el futuro.

Los datos anteriores señalan diferencias en el potencial de maltrato; en los que han sufrido maltrato físico y emocional, es mayor el porcentaje de sujetos con potencial de maltrato que reportan recuerdos de maltrato emocional que maltrato físico, lo mismo se repite en los sujetos que no reportan potencial de maltrato. Estos resultados nos orientan a reflexionar sobre el impacto psicológico diferenciado en ambos tipos de maltrato, considerando que, 
culturalmente, se asume que el maltrato físico es parte de la educación a los hijos, en cambio el maltrato emocional afecta la integridad yoica de la persona, vulnera sus seguridades y genera resentimientos que se mantienen latentes a lo largo de su historia personal.

Respecto a la relación de maltrato físico en la generación de padres e hijos, los hijos con recuerdos de maltrato físico tienen padres que también reportan recuerdos de maltrato físico $(64,4 \%)$ frente a un $35,6 \%$ cuyos padres no presentan recuerdos de maltrato físico; en la generación de hijos que no presentan recuerdos de maltrato físico en un $73 \%$ tiene padres que tampoco han sufrido maltrato físico y un $27 \%$ tiene padres que sí han sufrido maltrato físico

Los hijos con recuerdos de maltrato emocional tienen padres que a su vez reportan recuerdos de maltrato $(62,7 \%)$ y un $37,2 \%$ de padres que no han sufrido maltrato emocional, sin embargo han maltratado a sus hijos.

En el grupo de hijos sin recuerdos de maltrato, tienen padres $(29,1 \%)$ que habiendo sido maltratados no lo hacen con sus hijos.

Los hijos con recuerdos de abuso sexual tienen padres que a su vez registran abuso sexual, y padres que no han tenido recuerdos de abuso sexual, sin embargo, abusan de los hijos; igualmente, se puede observar que los hijos sin recuerdos de abuso sexual tienen padres que si han sufrido dicho abuso.

En el conjunto de resultados que analizan retrospectivamente la situación de maltrato, se encuentra una tendencia mayor de padres que habiendo sufrido algún tipo de maltrato: físico, emocional y abuso sexual también lo hacen con sus hijos, al lado de ello se encuentran padres que habiendo sido víctimas de alguna forma de maltrato, sin embargo, no lo hacen con sus hijos; de otro lado padres que no han sufrido maltrato y, sin embargo, maltratan a sus hijos.

Lo anterior nos orienta a afirmar que la reproducción generacional del maltrato no es absoluta y que más bien estarían presentes otras variables psicológicas, que hacen que por lo menos una tercera parte de padres maltratados no reproduzca el maltrato y otra parte importante de padres que, sin haber sido maltratados, si lo hacen con sus hijos, igualmente los hijos sin historia de maltrato presenten potencial de maltrato.

En un país como el nuestro en el que el maltrato es un problema de salud pública, es importante profundizar en el estudio de otras variables psicológicas asociadas al maltrato para favorecer estrategias de intervención y prevención más eficaces.

\section{CONCLUSIONES}

1. Existe una mayor incidencia del maltrato emocional en la generación de padres e hijos, seguida por el castigo físico.

2. En la generación de hijos se observa un descenso en el maltrato físico comparado con la generación de sus padres.

3. No existen diferencias de género y los recuerdos de maltrato físico, emocional y abuso sexual en la generación de hijos

4. En la generación de hijos se presenta $21,8 \%$ de potencial de maltrato 
5. Existe relación significativa entre el potencial de maltrato y los recuerdos de maltrato físico, emocional y abuso sexual.

6. Existe relación significativa entre el maltrato de la generación de hijos y la historia de maltrato de sus padres.

\section{REFERENCIAS BIBLIOGRÁFICAS}

1. De Paúl, J., Arruabarrena, M. (2001). Manual de protección infantil. Madrid: Masson.

2. De Paúl, J.,Perez-Albeniz, Montes, P. (2002). Recuerdos de maltrato infantil en maltratadores y potencial de maltrato en víctimas de maltrato físico y abuso sexual. En Psicothema, 14, 53-62.

3. De Paúl, J., Arrabuena, M., Mugica, P. (1999). "Validación Española del child abuse potencial inventory". Estudios de psicología. 63-64, 55-72.

4. Gomez,E.; De Paúl, J. (2003). La trasmisión intergeneracional del maltrato físico infantil. Psicothema, 15, 452-457.

5. Gracia, E. (2002). El maltrato infantil en el contexto de la conducta parental. Percepción de padres e hijos. Psicothema 14, 274-279.

6. Yanes, J. y gonzales, R. (2000). Correlatos cognitivos asociados a la experiencia de violencia familiar. Psicothema 12, 41-48.

7. Zingraf, M., Leiter, J. y Johnsen,M.(1993). Child maltreatment and youthful problem behavior. Criminology, 31, 173-202). 